\title{
Inhibition of Regulatory Volume Decrease Enhances the Cytocidal Effect of Hypotonic Shock in Hepatocellular Carcinoma
}

\author{
Michihiro Kudou1 ${ }^{*}$, Atsushi Shiozaki ${ }^{\boxplus 凶 *}$, Toshiyuki Kosuga ${ }^{*}$, Daisuke Ichikawa1 ${ }^{1}$ Hirotaka Konishi ${ }^{1}$, Ryo \\ Morimura $^{1}$, Shuhei Komatsu ${ }^{1}$, Hisashi Ikoma ${ }^{1}$, Hitoshi Fujiwara ${ }^{1}$, Kazuma Okamoto ${ }^{1}$, Shigekuni Hosogi ${ }^{2}$, \\ Takashi Nakahari², Yoshinori Marunaka2,3, Eigo Otsuji ${ }^{1}$ \\ 1. Division of Digestive Surgery, Department of Surgery, Kyoto Prefectural University of Medicine, Kyoto, 602-8566, Japan; \\ 2. Departments of Molecular Cell Physiology and Bio-Ionomics, Graduate School of Medical Science, Kyoto Prefectural University of Medicine, Kyoto, \\ 602-8566, Japan; \\ 3. Japan Institute for Food Education and Health, Heian Jogakuin (St. Agnes') University, Kyoto, 602-8013, Japan. \\ * These authors contributed equally to this work. \\ $\triangle$ Corresponding author: Dr. Atsushi Shiozaki, Assistant Professor, Division of Digestive Surgery, Department of Surgery, Kyoto Prefectural University of \\ Medicine, 465 Kajii-cho, Kamigyo-ku, Kyoto 602-8566, Japan. Tel.: +81 752515527, Fax: +81 752515522, e-mail: shiozaki@koto.kpu-m.ac.jp.
}

(1) Ivyspring International Publisher. Reproduction is permitted for personal, noncommercial use, provided that the article is in whole, unmodified, and properly cited. See http://ivyspring.com/terms for terms and conditions.

Received: 2016.02.03; Accepted: 2016.06.04; Published: 2016.07.08

\begin{abstract}
Background: Hypotonic shock induces cytocidal effects through cell rupture, and cancer therapy based on this mechanism has been clinically administered to hepatocellular carcinoma patients. We herein investigated the effectiveness of hypotonic shock combined with the inhibition of regulatory volume decrease as cancer therapy for hepatocellular carcinoma.

Methods: Morphological changes in human hepatocellular carcinoma cell lines were observed under a differential interference contrast microscope connected to a high-speed digital video camera. Cell volume changes under hypotonic shock with or without chloride, potassium, or water channel blockers were observed using a high-resolution flow cytometer. In order to investigate cytocidal effects, the number of surviving cells was compared after exposure to hypotonic solution with and without each channel blocker (re-incubation experiment).

Results: Video recordings showed that cells exposed to distilled water rapidly swelled and then ruptured. Cell volume measurements revealed regulatory volume decrease under mild hypotonic shock, whereas severe hypotonic shock increased the number of broken fragments as a result of cell rupture. Moreover, regulatory volume decrease was inhibited in cells treated with each channel blocker. Re-incubation experiments showed the cytocidal effects of hypotonic shock in cells exposed to hypotonic solution, and additional treatments with each channel blocker enhanced these effects.

Conclusion: The inhibition of regulatory volume decrease with chloride, potassium, or water channel blockers may enhance the cytocidal effects of hypotonic shock in hepatocellular carcinoma. Hypotonic shock combined with the inhibition of regulatory volume decrease was a more effective therapy than hypotonic shock alone.
\end{abstract}

Key words: Hepatocellular carcinoma, Regulatory volume decrease, hypotonic shock, potassium ion transport, chloride ion transport.

\section{Introduction}

Hepatocellular carcinoma (HCC) is a very common malignancy, and its estimated age-standardized mortality is the third highest worldwide [1]. Although recent advances in treatments directed at HCC patients have improved prognoses [2-3], those of HCC patients with spontaneous rupture have remained poor [4-5]. Previous studies identified HCC rupture as an 
independent risk factor for peritoneal metastasis in HCC patients [6-8], therefore, an effective therapy that prevents the development of peritoneal metastasis needs to be established.

Hypotonic shock is considered to induce cytocidal effects through cell rupture, and cancer therapy based on this mechanism is clinically administered to HCC patients, e.g. intraoperative peritoneal lavage with distilled water. This lavage procedure has been performed on digestive cancer patients, and has cytocidal effects on exfoliated viable cancer cells, thereby preventing peritoneal recurrence. We previously demonstrated the cytocidal effects of this therapy on esophageal, gastric, pancreatic and colorectal cancers in vitro and in vivo [9-14]. The efficacy of this therapy for HCC was reported previously, and has been employed during surgery [15-16]. However, we also showed that the osmolality of peritoneal lavage fluid collected from patients undergoing peritoneal lavage with distilled water increased to mild hypotonicity due to the contamination of disrupted cells [10]. Cancer cells often avoid rupture under mild hypotonicity through regulatory volume decrease (RVD), which is homeostatic volume regulation by water transport via the activation of chloride and potassium conductance, and the cytocidal effects of peritoneal lavage with distilled water may decline as a result of osmotic increases. Therefore, we considered it necessary to simultaneously regulate RVD in cancer cells in order to improve the efficacy of intraoperative peritoneal lavage with distilled water.

In the present study, we analyzed changes in cellular morphology and volume after hypotonic shock, and investigated the osmolality and incubation time needed to kill HCC cells using several unique methods, such as a differential interference contrast (DIC) microscope connected to a high-speed digital video camera, a high-resolution flow cytometer, and re-incubation experiments. We demonstrated that the cytocidal effects of hypotonic shock were enhanced in HCC cells under the inhibition of RVD by the chloride channel blocker, 5-nitro-2-(3-phenylpropylamino)benzoic acid (NPPB), potassium channel blocker, quinine hydrochloride (Quin), and water channel blocker, mercury chloride II (Hg). These results suggest that hypotonic shock combined with the inhibition of RVD is a more effective therapy than hypotonic shock alone.

\section{Materials and methods}

\section{Cell culture and materials}

The human HCC cell lines, HLE and Alexander, were obtained from the Japanese Collection of Research Bioresources Cell Bank. These cells, which had undergone less than thirty passages, were used in all analyses. They were grown in plastic culture flasks (Corning Incorporated, NY, USA); HLE cells were maintained in DMEM medium (Nacalai Tesque, Kyoto, Japan) and Alexander cells were maintained in RPMI-1640 medium (Nacalai Tesque, Kyoto, Japan). Each medium was supplemented with $10 \%$ fetal bovine serum (FBS), $100 \mathrm{U} / \mathrm{ml}$ of penicillin, and 100 $\mu \mathrm{g} / \mathrm{ml}$ of streptomycin. Flasks were kept in a humidified incubator at $37^{\circ} \mathrm{C}$ under $5.0 \% \mathrm{CO}_{2}$ in air. NPPB was purchased from BIOMOL International, L.P. (Plymouth Meeting, PA, USA). Quin and Hg were purchased from Nacalai Tesque.

\section{$\mathrm{NaCl}$ isotonic and hypotonic solutions}

The $140 \mathrm{mM} \mathrm{NaCl}$ isotonic solution contained $140 \mathrm{mM} \mathrm{NaCl}, 5.0 \mathrm{mM} \mathrm{KCl}, 1.0 \mathrm{mM} \mathrm{CaCl}, 1.0 \mathrm{mM}$ $\mathrm{MgCl} 2,5.0 \mathrm{mM}$ glucose, and $10 \mathrm{mM}$ HEPES. The $\mathrm{pH}$ of each solution was adjusted to 7.4 with $\mathrm{NaOH}$. In order to analyze changes in the volumes of cells subjected to hypotonic shock at various osmolarities, graded hypotonic $\mathrm{NaCl}$ solution was produced by diluting the stock $\mathrm{NaCl}$ solution with distilled water, for example, $1 / 4 \mathrm{NaCl}$ solution indicated $\mathrm{NaCl}$ solution diluted 4 -fold with distilled water.

\section{Observation of morphological changes in HCC cells after their exposure to distilled water}

HCC cells were washed with PBS and then detached from the flask using trypsin-EDTA. These cells were subsequently transferred to a centrifuge tube and centrifuged at $800 \mathrm{rpm}$ for $5 \mathrm{~min}$. The supernatant was then discarded, isotonic $\mathrm{NaCl}$ solution was added to the pelleted cells, and the suspended cells were mounted on a coverslip that had been pre-coated with neutralized Cell-Tak (Becton-Dickinson Labware, Bedford, MA, USA) in order to retain them firmly. The coverslip was then placed in a perfusion chamber that had been mounted on the stage of a DIC microscope (ECLIPSE Ti, Nikon, Japan) connected to a video-enhanced contrast (VEC) system (ARGUS-20, Hamamatsu Photonics, Hamamatsu, Japan), and images were continuously recorded by a video recorder, according to a previously described procedure [9-12]. Experiments were carried out at room temperature $\left(20-24^{\circ} \mathrm{C}\right)$. The volume of the perfusion chamber was approximately $20 \mu \mathrm{l}$, and the rate of perfusion was $200 \mu \mathrm{l} / \mathrm{min}$. The isotonic $\mathrm{NaCl}$ solution was initially perfused through the chamber, then replaced with distilled water, and morphological changes in HCC cells were observed. 


\section{Measurement of individual cell volume changes in HCC cells after exposure to distilled water using a microscope}

The method of individual cell volume measurements has been described previously [17-19]. During experiments, a movie of morphological changes was recorded using a DIC microscope connected to a high-speed digital video camera. The area of single HCC cells was measured from the recording every 30 seconds from the start of the experiments to 10 minutes later using DIPP-Motion, which is $2 \mathrm{D}$ motion capture software. The area of HCC cells before the start of the experiment was used as the control value $\left(\mathrm{A}_{0}\right)$. The relative volume of a HCC cell was expressed as $\mathrm{V} / \mathrm{V}_{0}\left[=\left(\mathrm{A} / \mathrm{A}_{0}\right)^{1.5}\right]$, where $\mathrm{V}$ is the volume, $\mathrm{A}$ is the area, and subscript 0 indicates the control value. The values of $V / V_{0}$ from four experiments were expressed as the mean \pm SEM.

\section{Measurement of cell volume changes in HCC cells after hypotonic shock using a high resolution flow cytometer}

Cell volume measurements were performed using a high resolution flow cytometer, the Cell Lab Quanta (Beckman Coulter, Fullerton, CA, USA), according to a previously described procedure [9-12]. This flow cytometer was designed to measure the electronic volume (EV) of a cell, and the EV data of more than 10,000 cells were collected and analyzed using Quanta control software. HCC cells grown in culture flasks were detached using trypsin-EDTA and centrifuged. A total of $1.0 \times 10^{6}$ pelleted cells were then suspended in $1 \mathrm{ml}$ of distilled water or hypotonic $\mathrm{NaCl}$ solution of various osmolarities to induce hypotonic shock. The suspended solution was subsequently displaced into a Vi-CELLTM Sample Cup (Beckman Coulter), and cell volume was measured 1, 5, 10, and $20 \mathrm{~min}$ after the exposure to each solution. The cell suspension in the isotonic $\mathrm{NaCl}$ solution was used as a control sample without hypotonic shock $(0 \mathrm{~min})$. In the chloride channel regulation experiments, cells were pre-incubated with culture medium containing $200 \mu \mathrm{M} \mathrm{NPPB}$ at $37^{\circ} \mathrm{C}$ for 30 min under $5.0 \% \mathrm{CO}_{2}$ in air. In the potassium channel regulation experiments, cells were suspended in $\mathrm{NaCl}$ solution containing $1 \mathrm{mM}$ Quin at room temperature for $15 \mathrm{~min}$ before hypotonic shock. A previous study reported that $\mathrm{Hg}$ inhibited RVD without a pre-treatment [23]; therefore, a pre-treatment was not performed to avoid cell toxicity by $\mathrm{Hg}$ in the water channel regulation experiments. In all regulation experiments, hypotonic solution containing each blocker: $200 \mu \mathrm{M}$ NPPB, $1 \mathrm{mM}$ Quin, or $2.5 \mu \mathrm{M} \mathrm{Hg}$, was used during hypotonic shock.

\section{Re-incubation of HCC cells after exposure to distilled water (DW re-incubation experiments)}

HCC cells grown in culture flasks were detached using trypsin-EDTA and centrifuged. A total of $2.0 \times 10^{5}$ pelleted HLE cells or $4.0 \times 10^{5}$ pelleted Alexander cells were then suspended in distilled water and incubated for 1, 5, 10, or $20 \mathrm{~min}$. Thereafter, the suspension was centrifuged, and the pelleted cells were re-suspended in culture medium and seeded on Costar 6-well plates (Corning Incorporated, NY, USA). At a set time of $48 \mathrm{~h}$ after plating, cells were detached from the plates in trypsin-EDTA solution, and a viable cell count was performed using Trypan blue and the Countess Automated Cell Counter (Invitrogen, Tokyo, Japan). The cell suspension in the isotonic $\mathrm{NaCl}$ solution was used as a control sample without hypotonic shock (0 min).

\section{Cell viability assay on adherent cells exposed to distilled water}

A total of $2.0 \times 10^{5}$ HLE or Alexander cells were seeded on 6-well plates. Medium was removed $24 \mathrm{~h}$ after plating, and distilled water was then applied to adherent HCC cells, which were incubated for 1, 5, 10, or $20 \mathrm{~min}$. After this treatment, distilled water was exchanged to medium containing FBS. At a set time of $48 \mathrm{~h}$ after the treatment, a viable cell count was performed using a similar method to the DW re-incubation experiment on suspended cells.

\section{Re-incubation of HCC cells after exposure to hypotonic solution under the regulation of channel activity (re-incubation experiments under channel regulation)}

In all channel regulation experiments, a similar pretreatment method to the measurement of cell volume changes was performed. The total cell number after re-incubation for $48 \mathrm{~h}$ after each treatment was counted by a similar method to that used in the DW re-incubation experiments. The relative cell number (total cell number/total cell number at $0 \mathrm{~min}$ ) was assessed at the indicated time after exposure to the hypotonic solution containing a channel blocker: 200 $\mu \mathrm{M} \mathrm{NPPB}, 1 \mathrm{mM}$ Quin, or $1 \mu \mathrm{M} \mathrm{Hg}$, or control hypotonic solution. In the chloride channel regulation experiments, $1 / 8 \mathrm{NaCl}$ solution was used as the hypotonic solution for Alexander cells, while 1/6 $\mathrm{NaCl}$ solution was used for HLE cells. Hypotonic solution containing $0.2 \%$ DMSO was used as the control solution. In the potassium channel regulation experiments, $1 / 8 \mathrm{NaCl}$ solution was used as the hypotonic solution for Alexander cells, while 1/4 $\mathrm{NaCl}$ solution was used for HLE cells. In the water channel regulation experiments, $1 / 4 \mathrm{NaCl}$ solution 
was used as the hypotonic solution for Alexander cells, while $1 / 6 \mathrm{NaCl}$ solution was used for HLE cells.

\section{Statistical analysis}

Results were expressed as means \pm SEM. Statistical analyses were carried out using the Student's $t$-test. Differences were considered significant when the $\mathrm{p}$-value was $<0.05$. Statistical analyses were performed using JMP version 10 .

\section{Results}

\section{Morphological changes in individual HCC cells after exposure to distilled water}

Morphological changes were observed in HCC cells after exposure to distilled water using a DIC microscope connected to a VEC system. HCC cells started to swell as soon as they were exposed to

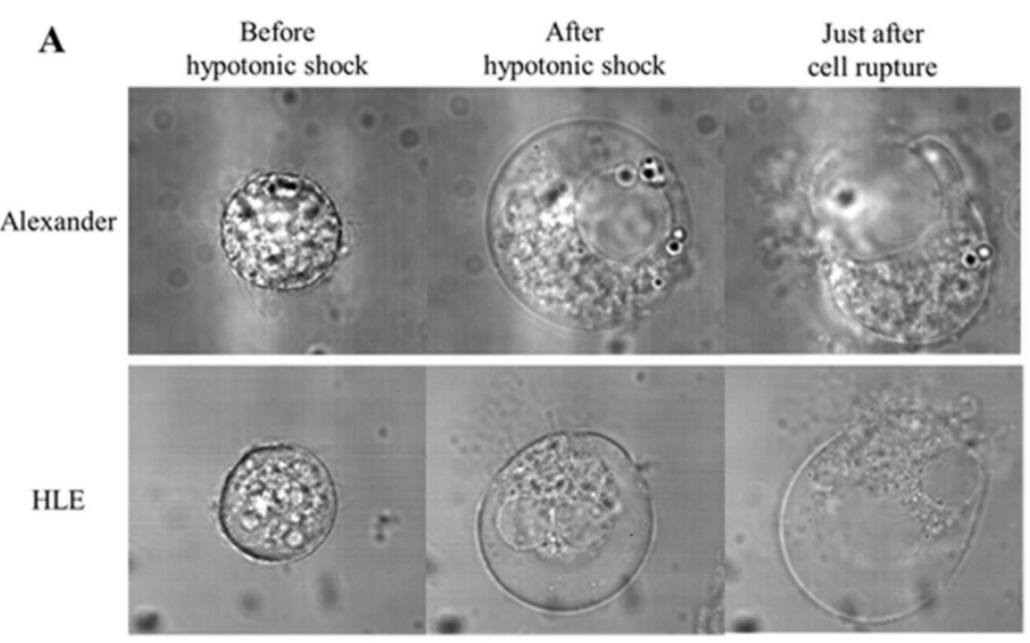

B

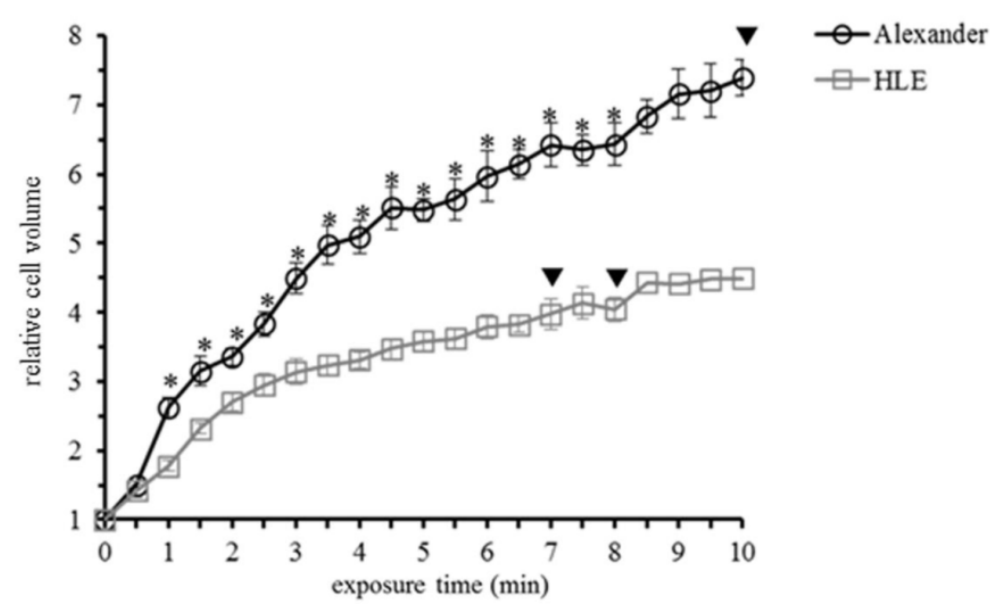

Figure 1. Morphological changes in individual HCC cells after hypotonic shock induced by distilled water. (A), Representative images of HLE and Alexander cells before and after hypotonic shock induced by distilled water. (B), Cell volume changes in individual HCC cells after hypotonic shock induced by distilled water. Cell volumes were measured every thirty seconds from the start of the exposure to distilled water to 10 minutes later. Results are presented as the mean \pm SEM $(n=4)$. The inverted triangle indicates that one out of four cells ruptured at this time point. ${ }^{*} \mathrm{p}<0.05$, significant difference between each cell line at the same time point. distilled water, and continued to swell until they ruptured. Alexander cells ruptured $567.2 \pm 64.4 \mathrm{sec}$ $(\mathrm{n}=17$, mean \pm SEM) after being exposed to distilled water, while HLE cells ruptured after $449.5 \pm 53.3 \mathrm{sec}$ $(\mathrm{n}=13$, mean \pm SEM) (Fig. 1A). Moreover, volume changes in four individual cells were measured for 10 minutes (Fig. 1B). The cell volume of Alexander cells increased 7-fold in 10 minutes, and one of the four cells ruptured 10 minutes after being exposed to distilled water. The volume of HLE cells increased 4-fold in 10 minutes, and two out of the four HLE cells ruptured 7 minutes and 8 minutes after being exposed to distilled water. The capacity of a cell to increase in volume was larger in Alexander cells than in HLE cells. Moreover, the time required for cell rupture was longer in Alexander cells than in HLE cells.

\section{Cell volume changes in HCC cells after hypotonic shock}

In order to analyze serial cell volume changes in HCC cells after hypotonic shock at various osmolarities, we simultaneously assessed cell volumes and counts after hypotonic shock using Cell Lab Quanta. Figure 2 shows the results for Alexander (Fig. 2A) and HLE (Fig. 2B) cells before and after hypotonic shock at various osmolarities. During the exposure to mild hypotonic shock, cell volumes initially increased, which indicated cell swelling, and subsequently decreased gradually to the level of pre-hypotonic shock despite the continuous presence of extracellular hypotonic solution, which indicated RVD. During the exposure to severe hypotonic shock using distilled water or $1 / 16 \mathrm{NaCl}$ solution, cell volumes also initially increased and then markedly decreased 5 or 20 min after being exposed. Cell volumes at these points were smaller than the initial cell volumes in all HCC cell lines. These results suggest that most cells had ruptured and broken into small fragments. Moreover, a marked increase was observed in the number of broken fragments of HLE cells exposed to $1 / 8$ $\mathrm{NaCl}$ solution, whereas only a slight increase was observed in those of Alexander cells, suggesting that Alexander cells were more resistant to hypotonic shock than HLE cells. 
A

Alexander

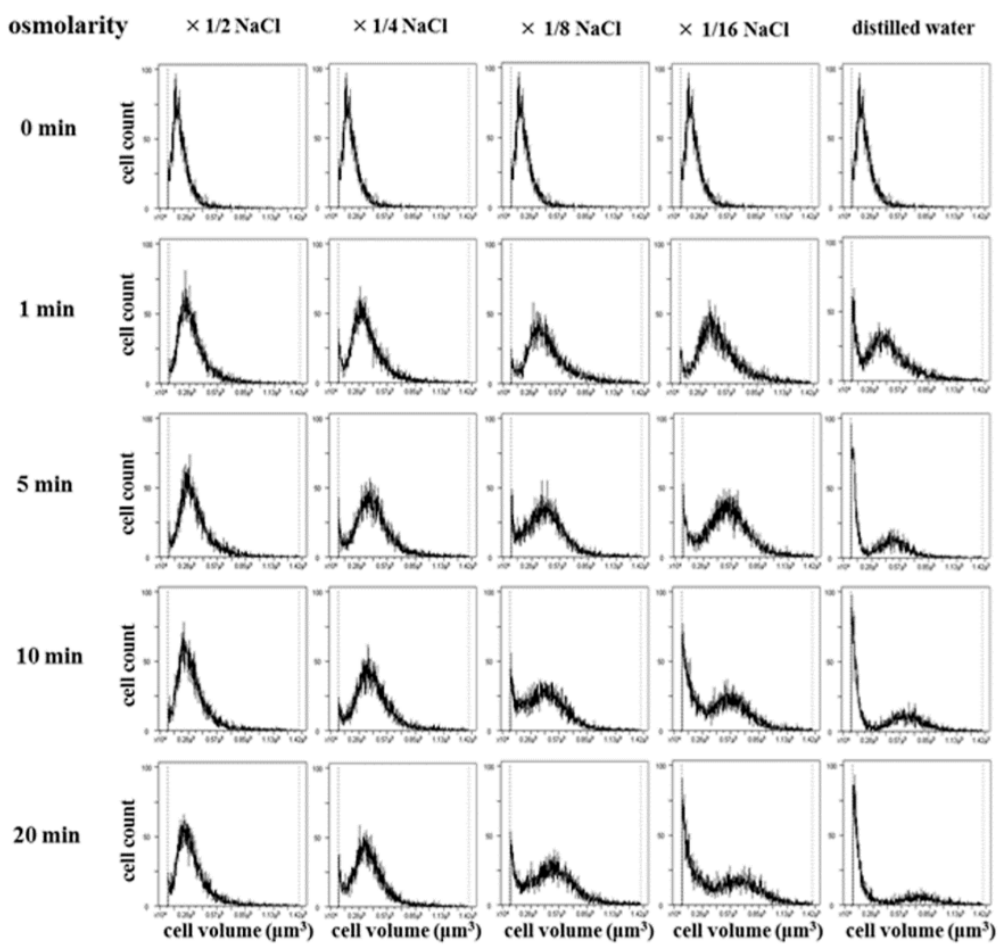

B

HLE
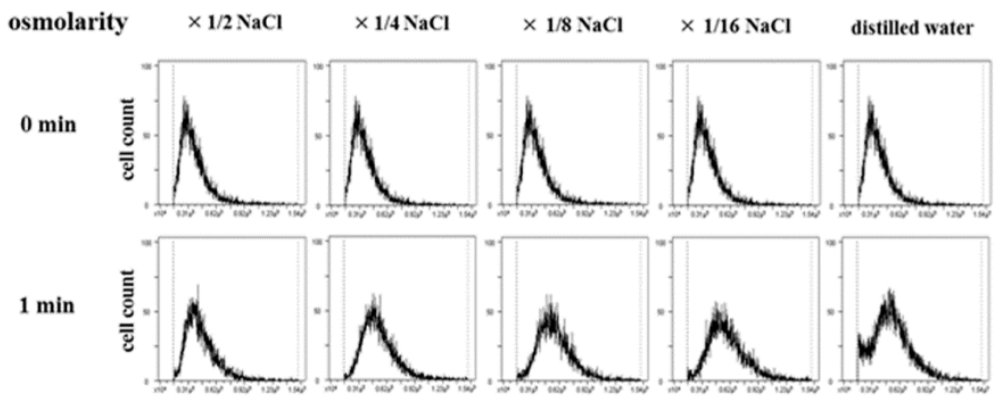

5 min
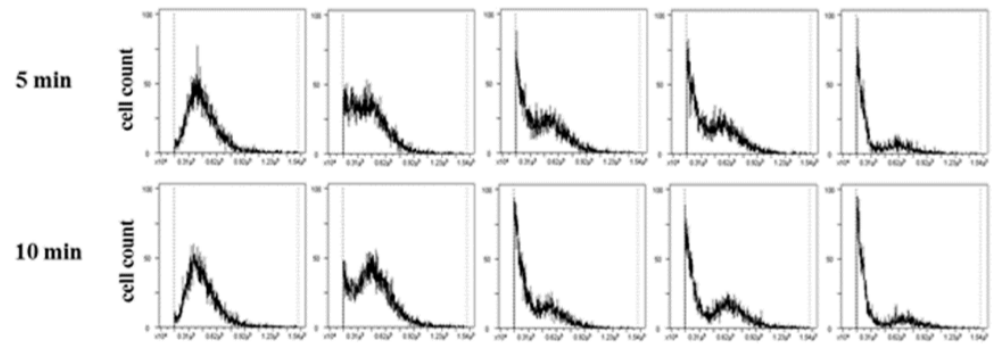

$20 \mathrm{~min}$
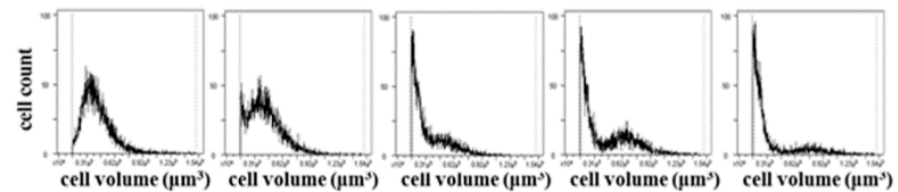

Figure 2. Cell volume changes in HCC cell populations after hypotonic shock. The cell volumes and cell counts of Alexander (A) and HLE (B) cells were simultaneously assessed 1, 5, 10, and 20 min after hypotonic shock at various osmolarities using a high-resolution flow cytometer, the Cell Lab Quanta. Isotonic $\mathrm{NaCl}$ solution was diluted 2-, 4-, 8-, and 16-fold with distilled water. A cell suspension in isotonic $\mathrm{NaCl}$ solution was used as a control (0 min).

\section{Cytocidal effects of distilled water on HCC cells (DW re-incubation experiments)}

In order to confirm the cytocidal effects of hypotonic shock induced by distilled water on HCC cancer cells, suspended HCC cell lines were re-incubated following their exposure to distilled water, and the cultured cell number was counted 48 hours later. The decreases observed in the number of HCC cells were dependent on the exposure time to 
distilled water (Fig. 3A). Few cells in each HCC cell line survived $48 \mathrm{~h}$ after a 10-min exposure to distilled water. The number of surviving cells was larger in Alexander cells exposed to distilled water for 1 or 5 min than in HLE cells. This result suggests differences in resistance to the cytocidal effects of hypotonic shock between each cell line, similar to cell volume changes in cell populations after hypotonic shock.

Furthermore, we investigated the cytocidal effects of distilled water on adherent HCC cells without the trypsin-EDTA treatment before hypotonic shock because this treatment may substantially change cell membrane structures and cell surface adhesion molecules, which may consequently result in artificially elevated sensitivity to hypotonic shock. The results obtained showed that the cytocidal effects of distilled water on adherent HCC cells were similar to those observed on suspended cells (Fig. 3B, 3C). Therefore, the extent of the cytocidal effects exerted by distilled water was similar in adherent and suspended HCC cells.
A

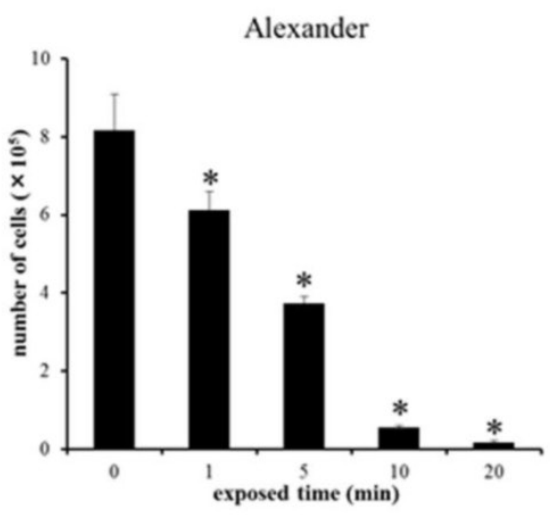

B

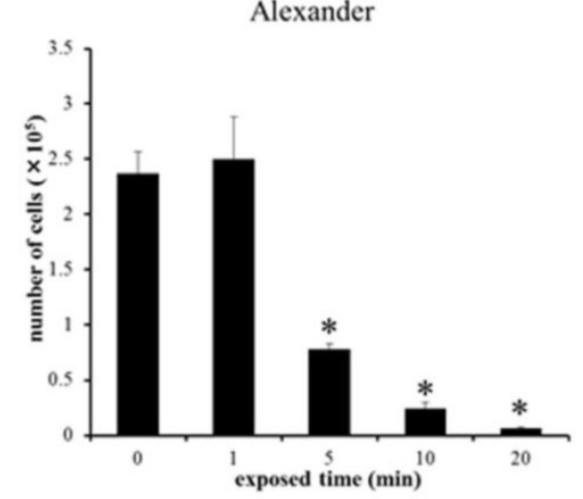

HLE

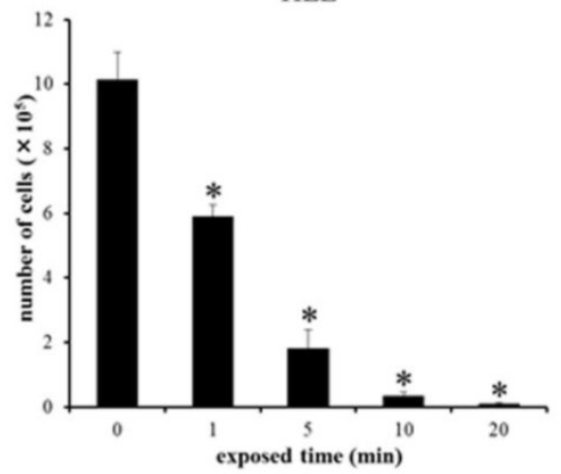

HLE

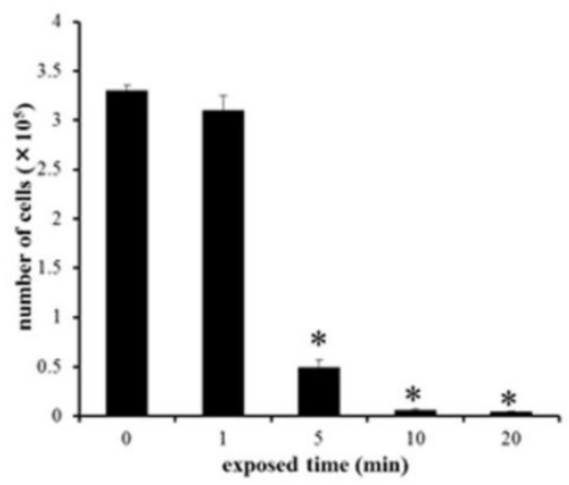

C
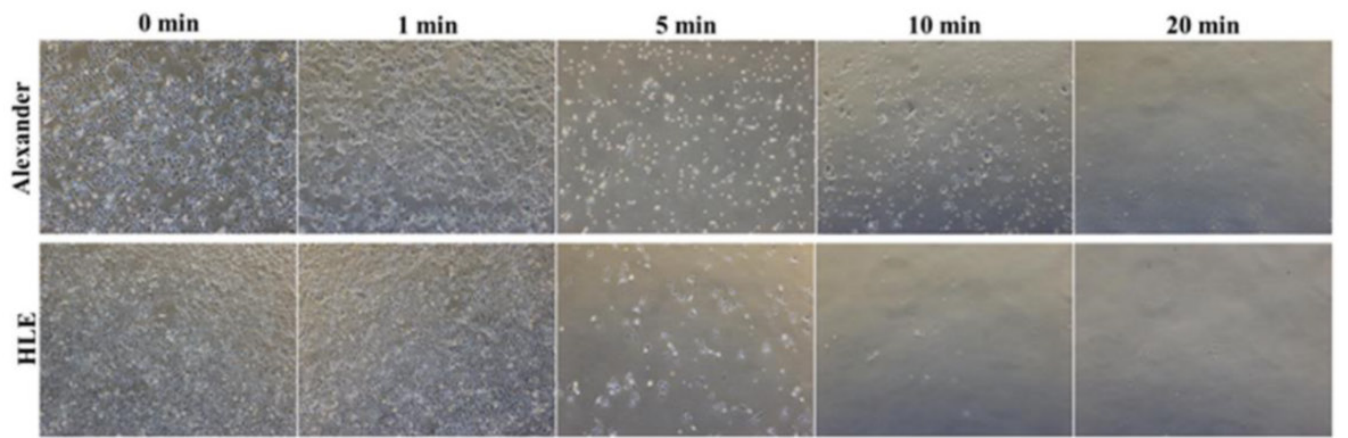

Figure 3. Re-incubation experiments on suspended $\mathrm{HCC}$ cells (A) and a cell viability assay on adherent $\mathrm{HCC}$ cells (B) after exposure to distilled water for $1,5,10$, and $20 \mathrm{~min}$. The number of cultured cells $48 \mathrm{~h}$ after exposure to distilled water was counted. Results are presented as the mean \pm SEM $(n=4)$. * $p<0.05$, significantly different from 0 min. (C), Representative images of cultured cells $48 \mathrm{~h}$ after adherent $\mathrm{HCC}$ cells were exposed to distilled water for the indicated time. 


\section{Inhibition of RVD by the blockade of chloride channels, potassium channels, and water channels affected cell volume changes in HCC cells}

We previously reported that the blockade of chloride ion transport inhibited RVD using NPPB in esophageal, gastric, pancreatic, and colorectal cancer cells [9-12]. Furthermore, RVD was shown to be inhibited by Quin or $\mathrm{Hg}$ through the blockade of potassium ion or water transport [20-23]. In order to investigate whether the regulation of ion and water transport affected cell volume changes in HCC cells after hypotonic shock, mean cell volumes were measured following exposure to the hypotonic $\mathrm{NaCl}$ solution with or without NPPB (DMSO was used as a control), Quin, or Hg using Cell Lab Quanta. Serial changes in the mean cell volume of HCC cells following their exposure to hypotonic solutions with or without each blocker are shown in Figure 5A-C. The cell volume of HCC cells treated with control hypotonic solution initially increased for $5 \mathrm{~min}$, and subsequently decreased from $10 \mathrm{~min}$ after the start of this treatment, which indicated RVD. In contrast, the cell volume of HCC cells treated with hypotonic solution containing NPPB, Quin, or $\mathrm{Hg}$ increased for 5 min, but did not subsequently decrease 10 min after the start of this treatment. These results suggest that each channel blocker inhibited RVD in HCC cell lines.
A

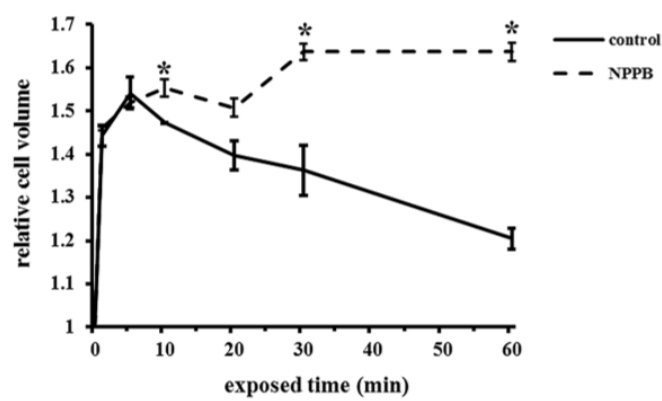

B

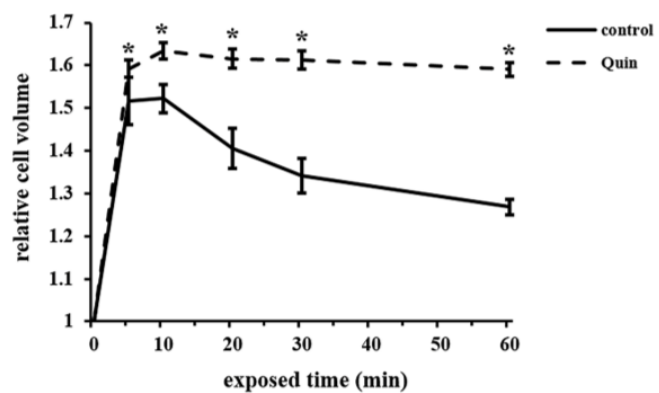

C

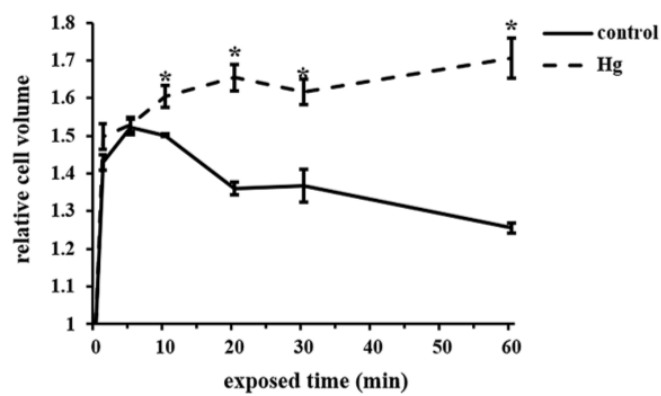

HLE

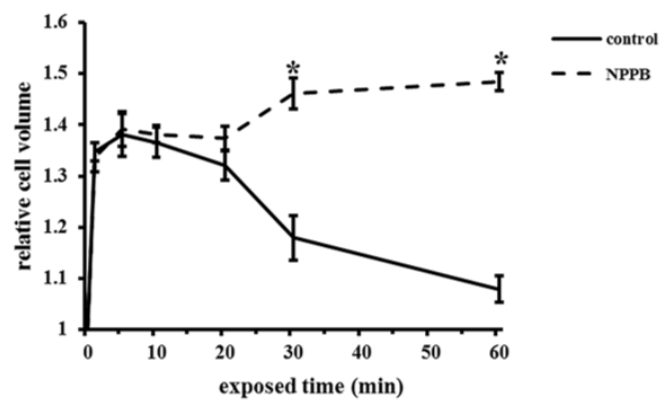

HLE

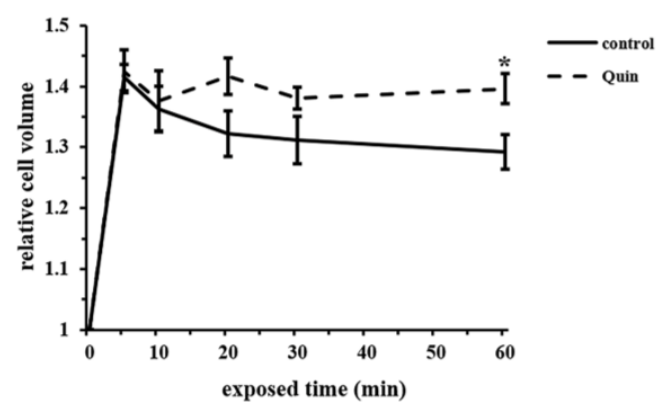

HLE

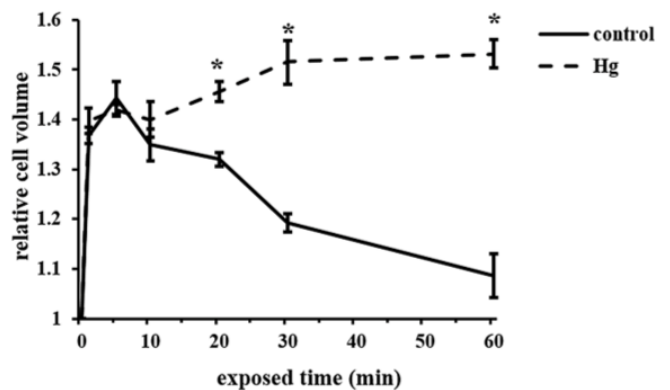

Figure 4. Effects of channel blockers on hypotonicity-induced cell volume changes in HCC cells. (A) Cell volume was measured at the indicated time after exposure to $1 / 2 \mathrm{NaCl}$ solution containing $200 \mu \mathrm{M}$ NPPB or $0.2 \%$ DMSO (control). The cell suspension in isotonic $\mathrm{NaCl}$ solution containing NPPB or DMSO was used as a sample without hypotonic shock $(0 \mathrm{~min})$. (B) Cell volume was measured after exposure to $1 / 2 \mathrm{NaCl}$ solution with $1 \mathrm{mM}$ quinine hydrochloride (Quin) and non-treated $1 / 2 \mathrm{NaCl}$ solution (control). The cell suspension in isotonic $\mathrm{NaCl}$ solution with or without $1 \mathrm{mM}$ Quin was used as a sample without hypotonic shock (0 min). (C) Cell volume was measured after exposure to $1 / 2 \mathrm{NaCl}$ solution with $2.5 \mu \mathrm{M}$ mercury chloride II $(\mathrm{Hg})$ and non-treated $1 / 2 \mathrm{NaCl}$ solution (control). The cell suspension in isotonic $\mathrm{NaCl}$ solution with or without $2.5 \mu \mathrm{M} \mathrm{Hg}$ was used as a sample without hypotonic shock (0 min). Results are presented as the mean \pm SEM $(n=3-5)$. ${ }^{*}<0.05$, significant difference from the control at the same time point. 


\section{Cytocidal effects of hypotonic shock on HCC cells were induced by the inhibition of RVD (re-incubation experiments under channel regulation)}

In order to investigate whether the cytocidal effects of mild hypotonic shock on HCC cells were induced by the inhibition of RVD, HCC cells exposed to hypotonic solution containing NPPB, Quin, or $\mathrm{Hg}$ were re-incubated, and the number of these cultured cells was counted 48 hours after the treatment.

Previous studies reported that NPPB, Quin, and $\mathrm{Hg}$ induced cytotoxicity and adverse events [24-26]. Therefore, we examined HCC cells treated with A

Alexander

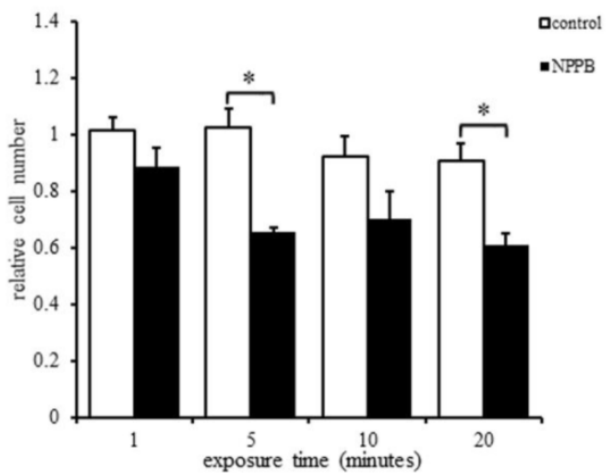

B

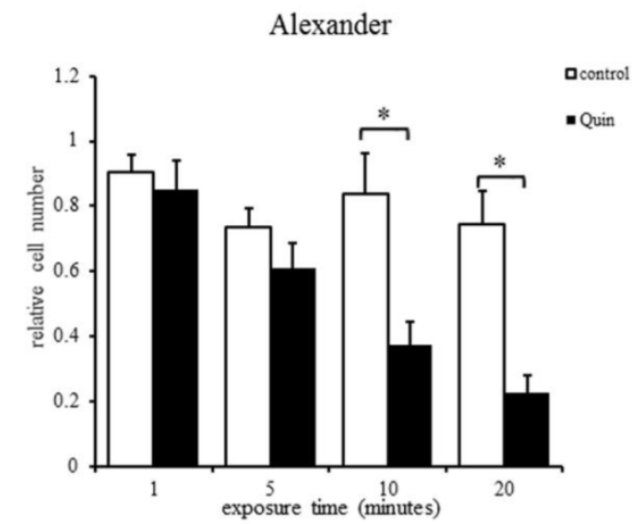

C

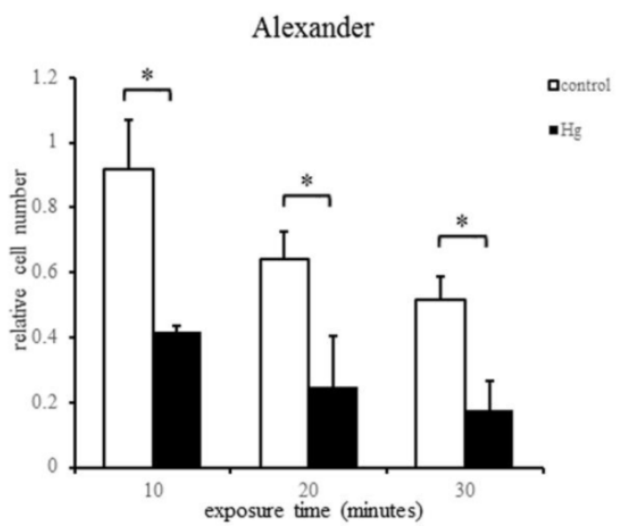

isotonic $\mathrm{NaCl}$ solution containing each blocker. No cellular toxicity or cytocidal effects were observed in HCC cells treated with isotonic solution containing each channel blocker (data are not shown).

Figure 5A shows the relative number of surviving cells after their exposure to the hypotonic $\mathrm{NaCl}$ solution with DMSO (control) or NPPB. Enhanced cytocidal effects were observed in HCC cells exposed to hypotonic solution containing NPPB. In the potassium channel regulation experiments, cytocidal effects were enhanced more in HCC cells exposed to Quin than in those exposed to the control (Fig. 5B). In the water channel regulation experiments, enhanced cytocidal effects were observed on HCC cells exposed to $\mathrm{Hg}$.
HLE

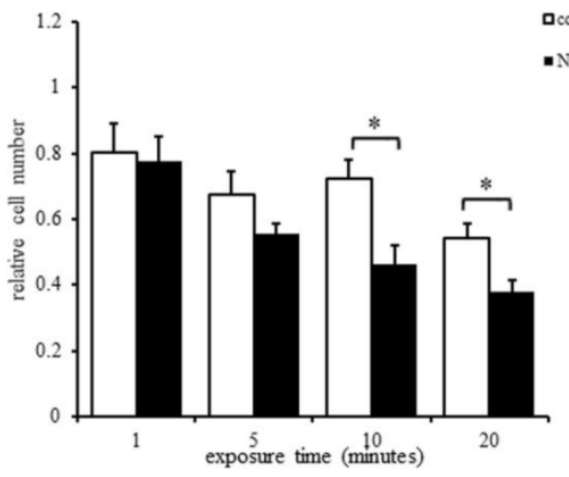

HLE

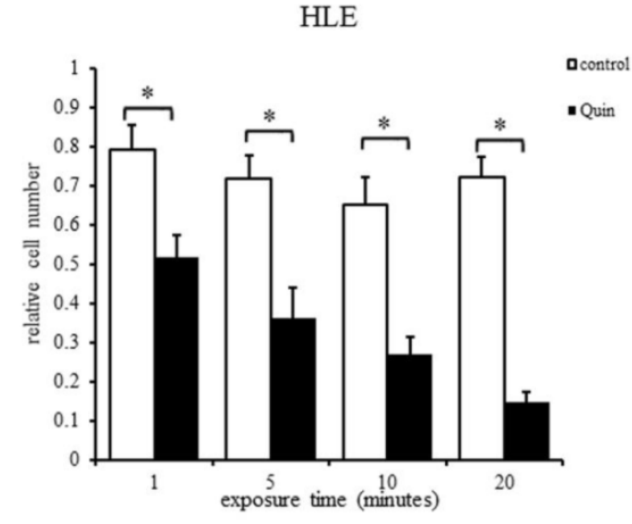

HLE

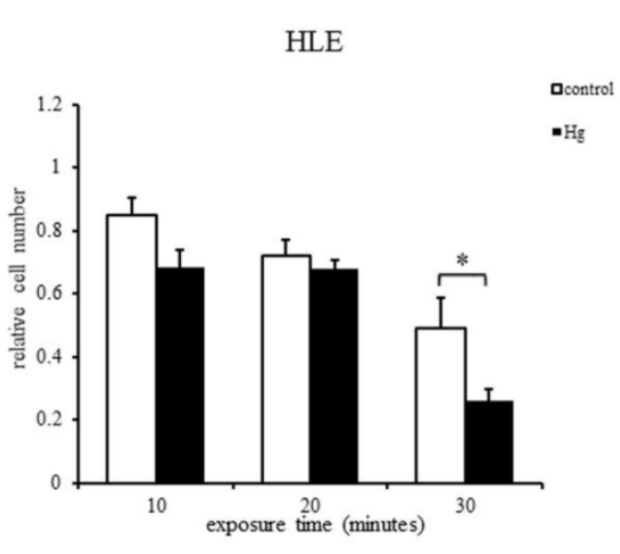

Figure 5. Re-incubation of $\mathrm{HCC}$ cells after their exposure to hypotonic solution containing each channel blocker: NPPB (A), Quin (B), or $\mathrm{Hg}(C)$. The relative cell number (total cell number/total cell number at $0 \mathrm{~min}$ ) was assessed at the indicated time after exposure to the hypotonic solution containing each channel blocker or control hypotonic solution (control). Results are presented as the mean $\pm \operatorname{SEM}(n=4)$. $*_{p}<0.05$, significant difference from the control at the same time point. 


\section{Discussion}

HCC is one of the most common cancers worldwide, with 782,451 new cases being reported in 2012 and approximately 745,533 HCC patients dying each year [1]. HCC is the second most common cause of death from cancer. In recent years, the pathogenic mechanism of HCC has been elucidated and various therapies have been developed for critical prevention and a complete cure. However, the prognosis of HCC patients has remained poor. The progression pattern of HCC cases is regarded as unique from other cancers, with distal, lymphatic, and peritoneal metastases being infrequently reported. In contrast, multiple primary cancers often synchronously or metachronously occur in the liver. However, a previous study showed that peritoneal metastasis often occurred in HCC cases with a previous history of tumor rupture [6-8, 27]. Exfoliated cancer cells have been suggested to spread in the abdominal cavity at the rupture of HCC tumors and implant onto the peritoneum, resulting in peritoneal metastasis [28]. Therefore, the prevention of peritoneal metastasis in HCC patients with tumor rupture is needed.

Peritoneal lavage with distilled water is regarded as an effective therapy for the prevention of peritoneal recurrence in spontaneously ruptured HCC. Previous studies reported that peritoneal lavage with distilled water significantly decreased tumor recurrence rates, and improved disease-free survival and overall survival in patients undergoing liver resection for spontaneously ruptured HCC $[15,16]$. However, we previously demonstrated that the osmolality of peritoneal lavage fluid collected from patients undergoing peritoneal lavage with distilled water was 54.7 mosmol $/ \mathrm{kg} / \mathrm{H}_{2} \mathrm{O}$ because of contamination by existing intraperitoneal secretions and the many cell types present in the peritoneal cavity [10], which may weaken the cytocidal effects induced by hypotonic shock. Under mild hypotonicity, human cells transiently swell and subsequently return to their original volume, thereby avoiding cell rupture. This phenomenon is generally called RVD, which is homeostatic cell volume regulation by water transport via the activation of potassium and chloride conductance. Hypotonic shock activates chloride and potassium channels (for example, VSOR and swelling-activated potassium channels) [29-31]. Therefore, the intracellular concentrations of potassium and chloride decrease, and intracellular water then moves to the extracellular side [29, 32, 33]. RVD may rescue exfoliated cells from rupture by peritoneal lavage with distilled water, leading to peritoneal recurrence. Therefore, we herein investigated whether the inhibition of RVD enhanced the cytocidal effects of mild hypotonic shock in HCC with the aim of establishing more effective peritoneal lavage to prevent peritoneal metastasis.

We observed morphological changes in individual HCC cells exposed to distilled water using a DIC microscope connected to a VEC system. HCC cells rapidly swelled and ruptured almost 10 minutes after the start of hypotonic shock. The volumes of these cells increased from 4-fold to 7-fold within 10 minutes (Fig. 1). A previous study reported that biological membranes have the ability to expand elastically by $3 \%$ before rupturing [34]; however, the cell surface area, which was calculated using DIC images, increased 2.5- to 4-fold in the present study. This may have been because the in-folded plasma membrane expanded, and more membrane was added from internal stores by cell surface area regulation (SAR) [35-36]. Furthermore, differences were observed in the capacity of Alexander and HLE cells to increase in volume. Volume measurements of cell populations using a flow cytometer and DW-reincubation experiments revealed that Alexander cells were more resistant to hypotonic shock than HLE cells. This result suggests that the capacity of cells to increase their volume is related to resistance to cytocidal effects and rupture by hypotonic shock.

We then observed cell volume changes in cancer cell populations exposed to hypotonic shock using a high resolution flow cytometer, the Cell Lab Quanta (Beckman Coulter, Fullerton, CA, USA). During exposure to severe hypotonic shock, increases in the number of small fragments were noted after cell swelling. These results suggest that most cells had ruptured and broken into fragments. RVD was observed during the exposure to mild hypotonic shock (Fig. 2). We previously demonstrated the inhibitory effects of RVD and enhanced cytocidal effects of hypotonic shock under the regulation of chloride ion movement with NPPB [9-12]. In the present study, potassium and water channel blockers were additionally employed in order to examine the importance of inhibiting RVD in peritoneal lavage with distilled water. A previous study reported that Quin and Hg inhibited RVD in other cell types [20-23], and we used these blockers as RVD inhibitors. We showed that RVD was clearly inhibited by these inhibitors, and the cytocidal effects of mild hypotonic shock in HCC cells treated with RVD inhibitors were enhanced in re-incubation experiments under channel regulation (Fig. 4, 5). Although the microenvironment of exfoliated cells is far more complex than laboratory settings, we considered the present results to show that exposure to hypotonic solution containing RVD inhibitors may be effective as a cancer treatment for HCC. However, NPPB, Quin, and Hg are toxic drugs 
that induce various organ disorders, and it is difficult to administer these drugs clinically [24-26]. Moreover, the safety of peritoneal lavage with distilled water has yet to be established; it has been suggested to induce water intoxication [37]. We previously reported that severe hypotonic shock induced cell rupture in human lung fibroblast WI38 cells. Furthermore, cancer cells were found to be more sensitive to hypotonic shock than fibroblast cells [11]. We also showed that all mice that underwent peritoneal lavage with distilled water survived, suggesting that distilled water did not cause fatal damage in vivo [12]. Therefore, peritoneal lavage with distilled water containing RVD inhibitors may be an effective therapy for preventing peritoneal metastasis in HCC patients with tumor rupture if safe RVD inhibitors are used.

In conclusion, we herein demonstrated the cytocidal effects of hypotonic shock induced by distilled water in HCC cells under the regulation of RVD with chloride, potassium, and water channel blockers. Hypotonic shock combined with the inhibition of RVD was a more effective therapy than hypotonic shock alone.

\section{Acknowledgments}

This work was supported by a Grant-in-Aid for Scientific Research (C) (26461988) from the Japan Society for the Promotion of Science.

\section{Competing Interests}

The authors have declared that no competing interest exists.

\section{References}

1. Torre L A, Bray F, Siegel R L, et al. Global cancer statistics, 2012. CA Cancer J Clin 2015; 65(2): 87-108.

2. Llovet J M, Burroughs A, Bruix J. Hepatocellular carcinoma. Lancet 2003; 362(9399): 1907-17.

3. Slotta J E, Kollmar O, Ellenrieder V, et al. Hepatocellular carcinoma: Surgeon's view on latest findings and future perspectives. World J Hepatol 2015; 7(9): $1168-83$

4. Aoki T, Kokudo N, Matsuyama Y, et al. Prognostic impact of spontaneous tumor rupture in patients with hepatocellular carcinoma: an analysis of 1160 cases from a nationwide survey. Ann Surg 2014; 259(3): 532-42.

5. Chua T C, Morris D L. Exploring the role of resection of extrahepatic metastases from hepatocellular carcinoma. Surg Oncol 2012; 21(2): 95-101.

6. Kwak M S, Lee J H, Yoon $\mathrm{J} \mathrm{H}$, et al. Risk factors, clinical features, and prognosis of the hepatocellular carcinoma with peritoneal metastasis. Dig Dis Sci 2012; 57(3): 813-9.

7. Gomaa A I, Waked I. Recent advances in multidisciplinary management of hepatocellular carcinoma. World J Hepatol 2015; 7(4): 673-87.

8. Matsukuma S, Sato K. Peritoneal seeding of hepatocellular carcinoma: clinicopathological characteristics of 17 autopsy cases. Pathol Int 2011; 61(6): 356-62.

9. Kosuga T, Shiozaki A, Ichikawa D, et al. Pleural lavage with distilled water during surgery for esophageal squamous cell carcinoma. Oncol Rep 2011; 26(3): 577-86.

10. Jitaka $D$, Shiozaki $A$, Ichikawa $D$, et al. Blockade of chloride ion transport enhances the cytocidal effect of hypotonic solution in gastric cancer cells. J Surg Res 2012; 176(2): 524-34.

11. Nako Y, Shiozaki A, Ichikawa D, et al. Enhancement of the cytocidal effects of hypotonic solution using a chloride channel blocker in pancreatic cancer cells. Pancreatology 2012; 12(5): 440-8.
12. Takemoto K, Shiozaki A, Ichikawa D, et al. Evaluation of the efficacy of peritoneal lavage with distilled water in colorectal cancer surgery: in vitro and in vivo study. J Gastroenterol 2015; 50(3): 287-97.

13. Shiozaki A, Ichikawa D, Takemoto $K$, et al. Efficacy of a hypotonic treatment for peritoneal dissemination from gastric cancer cells: an in vivo evaluation. Biomed Res Int 2014; 2014: 707089.

14. Shiozaki A, Ichikawa D, Otsuji E, et al. Cellular physiological approach for treatment of gastric cancer. World J Gastroenterol 2014; 20(33): 11560-6.

15. Chang Y M, Hsu K F, Yu J C, et al. Distilled water peritoneal lavage in patients with rupture hepatocellular carcinoma. Hepatogastroenterology 2013; 60(121): $140-3$

16. Zhou S J, Zhang E L, Liang B Y, et al. Distilled Water Lavage During Surgery Improves Long-Term Outcomes of Patients with Ruptured Hepatocellular Carcinoma. J Gastrointest Surg 2015; 19(7): 1262-70.

17. Nakahari T, Imai Y. Transient swelling of salivary acinus induced by acetylcholine stimulation: water secretion pathway in rat submandibular gland. J Membr Biol 1998; 161(3): 287-96.

18. Nakahari T, Murakami M, Kataoka T. Shrinkage of rat mandibular acinar cell with acetylcholine detected by video-enhanced contrast microscopy. Jpn J Physiol 1989; 39(4): 609-15.

19. Nakahari $\mathrm{T}$, Murakami $\mathrm{M}$, Yoshida $\mathrm{H}$, et al. Decrease in rat submandibular acinar cell volume during ACh stimulation. Am J Physiol 1990; 258(6 Pt 1): G878-86.

20. Miyazaki $\mathrm{H}$, Shiozaki A, Niisato $\mathrm{N}$, et al. Physiological significance of hypotonicity-induced regulatory volume decrease: reduction in intracellular $\mathrm{Cl}$ - concentration acting as an intracellular signaling. Am J Physiol Renal Physiol 2007; 292(5): F1411-7.

21. Caplanusi A, Kim K J, Lariviere E, et al. Swelling-activated K+ efflux and regulatory volume decrease efficiency in human bronchial epithelial cells. J Membr Biol 2006; 214(1): 33-41.

22. Ballatori N, Boyer J L. Disruption of cell volume regulation by mercuric chloride is mediated by an increase in sodium permeability and inhibition of an osmolyte channel in skate hepatocytes. Toxicol Appl Pharmacol 1996; 140(2): 404-10

23. Heo J, Meng F, Sachs F, et al. Dynamic effects of $\mathrm{Hg} 2+$-induced changes in cell volume. Cell Biochem Biophys 2008; 51(1): 21-32.

24. Van Vleet $T$ R, Schnellmann $R$ G. Toxic nephropathy: environmental chemicals. Semin Nephrol 2003; 23(5): 500-8.

25. Waalkes M P, Fox D A, States J C, et al.Metals and disorders of cell accumulation: modulation of apoptosis and cell proliferation. Toxicol Sci 2000; 56(2): 255-61.

26. Alkadi H O. Antimalarial drug toxicity: a review. Chemotherapy 2007; 53(6): 385-91.

27. Portolani N, Baiocchi G L, Gheza F, et al. Parietal and peritoneal localizations of hepatocellular carcinoma: is there a place for a curative surgery? World J Surg Oncol 2014; 12: 298

28. Jayne D. Molecular biology of peritoneal carcinomatosis. Cancer Treat Res 2007; 134: 21-33.

29. Hoffmann E K, Lambert I H, Pedersen S F. Physiology of cell volume regulation in vertebrates. Physiol Rev 2009; 89(1): 193-277.

30. Okada Y, Sato K, Numata T. Pathophysiology and puzzles of the volume-sensitive outwardly rectifying anion channel. J Physiol 2009; $587(\mathrm{Pt}$ 10): $2141-9$.

31. Pedersen S F, Klausen T K, Nilius B. The identification of a volume-regulated anion channel: an amazing Odyssey. Acta Physiol (Oxf) 2015; 213(4): 868-81.

32. Kida $\mathrm{H}$, Miyoshi $\mathrm{T}$, Manabe $\mathrm{K}$, et al. Roles of aquaporin-3 water channels in volume-regulatory water flow in a human epithelial cell line. J Membr Biol 2005; 208(1): 55-64.

33. Sardini A, Amey J S, Weylandt K-H, et al. Cell volume regulation and swelling-activated chloride channels. Biochimica et Biophysica Acta (BBA) Biomembranes 2003; 1618(2): 153-62.

34. Nichol J A, Hutter O F. Tensile strength and dilatational elasticity of giant sarcolemmal vesicles shed from rabbit muscle. J Physiol 1996; 493 ( Pt 1): $187-98$

35. Morris C E, Homann U. Cell surface area regulation and membrane tension. J Membr Biol 2001; 179(2): 79-102.

36. Wan X, Harris J A, Morris C E. Responses of neurons to extreme osmomechanical stress. J Membr Biol 1995; 145(1): 21-31.

37. MINAMI $Y$, ITO N, WATABE R, et al. Patient with pseudomyxoma peritonei in whom, hyperglycemia and hyponatremia were induced by massive intraperitoneal lavage with 5\% glucose. Masui 2012; 61(10): 1133-6. 